\title{
The optical reflector system for the CANGAROO-II imaging atmospheric Cherenkov telescope
}

\author{
A. Kawachi ${ }^{\text {a,1 }}$ Y. Hayami ${ }^{\text {b }}$, J. Jimbo ${ }^{\text {c }}$, S. Kamei ${ }^{\text {b }}$, T. Kifune ${ }^{\text {a }}$, \\ H. Kubo ${ }^{\text {b }}$, J. Kushida ${ }^{\text {b }}$, S. LeBohec ${ }^{\text {a,2 }}$, K. Miyawaki ${ }^{d}$, \\ M. Mori ${ }^{a}$, K. Nishijima ${ }^{c}$, J.R. Patterson ${ }^{\text {e }}$, R. Suzuki ${ }^{b}$, \\ T. Tanimori ${ }^{b}$, S. Yanagita ${ }^{f}$, T. Yoshikoshi ${ }^{a, 3}$, A. Yuki $^{g}$ \\ ${ }^{a}$ Institute for Cosmic Ray Research, University of Tokyo, Tanashi, Tokyo \\ 188-8502, Japan \\ ${ }^{\mathrm{b}}$ Department of Physics, Tokyo Institute of Technology, Meguro, Tokyo 152-8551, \\ Japan \\ ${ }^{\mathrm{c}}$ Department of Physics, Tokai University, Hiratsuka, Kanagawa 259-1292, Japan \\ ${ }^{\mathrm{d} C o m m u n i c a t i o n}$ Systems Center, Mitsubishi Electric Corporation, Amagasaki, \\ Hyogo 661-8661, Japan \\ ${ }^{\mathrm{e}}$ Department of Physics and Mathematical Physics, University of Adelaide, South \\ Australia 5005, Australia \\ ${ }^{\mathrm{f}}$ Faculty of Science, Ibaraki University, Mito, Ibaraki 310-8521, Japan \\ ${ }^{8}$ STE Laboratory, Nagoya University, Nagoya, Aichi 464-8601, Japan
}

\begin{abstract}
A new imaging atmospheric Cherenkov telescope with a light-weight reflector has been constructed. Light, robust, and durable mirror facets of containing CFRP (Carbon Fiber Reinforced Plastic) laminates were developed for the telescope. The reflector has a parabolic shape $(f / 1.1)$ with a $30 \mathrm{~m}^{2}$ surface area which consists of sixty spherical mirror facets. The image size of each mirror facet is $0^{\circ} .08$ (FWHM) on average. The attitude of each facet can be adjusted by stepping motors. After
\end{abstract}


the first in situ adjustment, a point image of about $0^{\circ} .14$ (FWHM) over 3 degree field-of-view was obtained. The effect of gravitational load on the optical system was confirmed to be negligible at the focal plane. The telescope has been in operation with an energy threshold for gamma-rays of $\lesssim 300 \mathrm{GeV}$ since May 1999 .

Key words: gamma-ray telescopes; ground-based instruments; imaging atmospheric Cherenkov technique; new instrument; reflector.

PACS: 95.55.Ka

\section{Introduction}

It is in the last decade that major discoveries and progress in the field of $\mathrm{TeV}$ gamma-ray astronomy have been achieved by using ground-based imaging atmospheric Cherenkov telescopes [1,2]. Currently, the lowest achieved energy threshold among the imaging Cherenkov telescopes is about $250 \mathrm{GeV}$. The experimental technique is considered to be still in its development phase, and we need improvements for the next generation of imaging atmospheric Cherenkov telescopes. At present, the number of reported $\mathrm{TeV}$ sources is about 10, while more than 250 sources are reported in the $100 \mathrm{MeV}$ to $30 \mathrm{GeV}$ band in the third EGRET catalogue [3]. So, a wealth of results is expected in the unexplored energy region near $100 \mathrm{GeV}$, and world-wide efforts are being made to lower the energy threshold of detectable gamma-rays down to $100 \mathrm{GeV}$ or even below so that the energy coverage overlaps with that of satellite detectors; for example, the STACEE [4] and CELESTE [5] experiments which have started operations using solar heliostats as large area photon collectors, the MAGIC project [6] for a high sensitivity telescope of a $17-\mathrm{m}$ diameter mirror,

$\overline{1}$ E-mail: kawachi@icrr.u-tokyo.ac.jp

2 present address: Department of Physics and Astronomy, Iowa State University, Ames, IA 50011-3160, U.S.A.

3 present address: Department of Physics, Osaka City University, Sumiyoshi-ku, Osaka 558-8585, Japan 
the VERITAS project [7] and the HESS project [8] for arrays of multiple imaging telescopes of 10-m aperture size. The CANGAROO project has studied TeV gamma-ray sources since 1992 , at Woomera, South Australia $\left(136^{\circ} \mathrm{E}, 31^{\circ}\right.$ S, $220 \mathrm{~m}$ a.s.l.) with a 3.8-m imaging atmospheric Cherenkov telescope $[9,10]$. In the second phase of the project (CANGAROO-II), the 3.8-m telescope has been replaced by a 7-m telescope located near the 3.8-m telescope site which started operation in May 1999 [11]. The third phase of the CANGAROO project (CANGAROO-III) has commenced, and an array of four 10-m telescopes will start observation in 2004 [10].

A typical imaging atmospheric Cherenkov telescope has a reflector to collect Cherenkov light from extensive air showers which develop in the upper atmosphere, and to focus the showers' images onto a multi-pixel camera. The images have an extended shape, governed by the Cherenkov angle and by multiple scattering of the charged shower components. The average Cherenkov photon density is almost proportional to the primary energy of the incident gammaray. Thus, a larger reflector can directly lower the observational gamma-ray energy threshold. Gamma-ray showers from a point-like source are discriminated from the overwhelming background of cosmic ray showers using differences in their images caused by their differing shower developments [12]. The analyses of the image shapes of the showers with an accuracy of $0^{\circ} .1-0^{\circ} .2$ has led to the success of the imaging Cherenkov technique in $\mathrm{TeV}$ gamma-ray detection ([2] and references therein). Within the constraints of engineering, fabrication, and assembly costs, telescopes are usually limited to diameters of $10 \mathrm{~m}$, and the large light collection area is obtained by tessellated multiple mirror facets of diameter less than $1 \mathrm{~m}$. As the reflector size increases, the gravitational load on the optical system may cause pointing deviations or deformation of images at the focal plane during observations. The optimum design has to attempt to minimize the effect of gravity within the other constraints on the design and construction.

For the 7-m reflector, we have developed a new type of mirrors based on CFRP (Carbon Fiber Reinforced Plastic) laminate. Our trial for mirrors of 
composite materials like CFRP encountered some difficulties in their realization; production of a composite mirror facet of different materials with good accuracies requires delicate control over many parameters, e.g. slight changes of autoclave temperature as a function of time. Nevertheless, the merit of a CFRP laminate mirror is that it is light, robust, and durable. After developing the CFRP-based mirror, we have been able to construct a large but light-weight and easy to handle optical system almost free from the effect of gravity, at reasonable cost and with manageable construction labor. We also employed computer-controlled stepping motors for on-site alignment of each mirror facet which enables us to obtain the best focus of the 7-m tessellated reflector. The telescope is also a prototype for, and will be the first telescope of, the CANGAROO-III project.

In this paper, we describe the design, manufacturing, alignment procedure, and the performance of the CANGAROO-II optical reflector system, expanding upon the preliminary descriptions given elsewhere [13]. All of these techniques provide us with useful knowledge to prepare for final design of the CANGAROO-III telescope as well as of even larger Cherenkov telescopes for the future.

\section{Design of the 7-m Telescope}

Figure 1 shows a view of the reflector and the camera support of the telescope. The reflector is an $f / 1.1$ tessellated paraboloid with a diameter of $7 \mathrm{~m}$, and with an effective light collecting area of $30 \mathrm{~m}^{2}$. Each of the sixty mirror facets is spherical in shape, and has an $0.8 \mathrm{~m}$ diameter and a radius of curvature of $16.4 \mathrm{~m}$ (on average). The prime focal plane of the reflector is equipped with a camera of 512 fast photo-multiplier tubes (PMTs) of $13 \mathrm{~mm}$ diameter with UV-glass (Hamamatsu, R4124UV). The PMTs are arranged with a spacing of $16 \mathrm{~mm}\left(0^{\circ} .115\right)$ to cover a field-of-view of about $3^{\circ}$, and are supplemented by $16 \mathrm{~mm} \times 16 \mathrm{~mm}$ light-collecting cones to reduce the dead space between 
photosensitive area of the PMTs.

\subsection{Mechanical Structure}

The reflector trusses were based on the technique of commercially available radio communication antennas ${ }^{4}$, and the nine honeycomb panels are mounted on the trusses. Six to nine mirror facets were installed on each honeycomb panel. The rough alignment of the mirror facets was performed at the factory for each panel, which were then shipped with the facets installed. The camera support has a simple mechanical structure where the four camera stays are connected directly to the center ring to hold the camera. The present support frame allows us to extend the reflector to $10-\mathrm{m}$ diameter by the additional of 54 more mirror facets early in the year 2000 .

The total weight of the moving part of the $7-\mathrm{m}$ telescope is 6.3 ton, similar to that of of the 6 ton CAT telescope [14]. However, the 7-m telescope has almost twice the effective reflector area of the CAT telescope. The light-weight but robust structure was designed to be able to be operated in winds of average velocity $30 \mathrm{~km} / \mathrm{hr}$ and operational for gusts up to $100 \mathrm{~km} / \mathrm{hr}$. The load of the camera and its cables is about $100 \mathrm{~kg}$ at the $8-\mathrm{m}$ focal length. Gravitational deflections of the camera support are a potential cause of focal point shifting, however the deviation in the pointing accuracy at all the elevation angles was measured to be less than $1^{\prime}$ (the nominal value). These measurements will be presented in more detail in Section 6.

\subsection{Optical Parameters}

Gamma-ray showers can be preferentially selected over accidental events due to the night sky background by the use of a narrow timing gate of $10 \mathrm{~ns}$ or so [15]. For good timing information, we chose a paraboloid design which

$\overline{4}$ Mitsubishi Electric Corporation, Communication Systems Center 
provides isochronous collection of photons. The alternative design used in a number of other imaging atmospheric Cherenkov telescopes (for example, see [16]) is the Davies-Cotton type of reflector where identical spherical mirror facets are mounted on a spherical structure with a radius of curvature that is exactly half that of the facets [17]. The maximum variation in photon arrival times from different portions of a Davies-Cotton type $(f / 0.7)$ 10-m reflector is 6 ns [16], while the variation calculated with our tessellated paraboloid design is less than $0.2 \mathrm{~ns}$, even for an extended diameter of $10 \mathrm{~m}$.

Another important point in the optical designing was the off-axis focusing ability; it is desirable to minimize the smearing of the point spread function and resulting off-axis decrease in the light concentration. Generally, a spherical reflector has better off-axis properties than a paraboloid, and the Davies-Cotton design has an advantage over the paraboloid design for Cherenkov imaging telescopes which require a wide field-of-view (usually more than 3 degrees). When appropriate radii of curvature of the facets are chosen, a parabolic tessellated reflector can achieve acceptable off-axis performance, especially in the light concentration. As the result of a ray-tracing calculation, for a 7-m reflector of sixty spherical mirror facets, a $16.4 \mathrm{~m}$ radius of curvature gives the best performance when all facets have identical curvature radii [15]. An arrangement of the mirror facets of slightly differing radii of curvature improves the performance further. There is a variance in the radii of curvature of the CFRP composites in the manufacturing process (Figure 4) and we took advantage of this by arranging the facets based on the radii of curvature referring to the results of the ray-tracing program.

Assuming the point spread function of a facet $\left(\sigma=0^{\circ} .1\right)$, the light concentration within one camera pixel $\left(0^{\circ} .115\right.$ square $)$ was calculated by a ray-tracing program with our design parameters, and with the Davies-Cotton design of the same $f$-number, of the same size and number of facets. The centers of off-axis images are shifted on the focal plane by the combined effect of the blurs of the facets and their off-axis aberrations. The ray-tracing calculations estimated the peak-point shifts of about $5 \%$ (our design) and of $1 \%$ (the Davies-Cotton 
design) at the edge of our field-of-view ( $1^{\circ} .3$ off the axis). On-axis and at the edge of the field-of-view, the portions of photons which fall within a camera pixel at the image center are estimated to be $59 \%$ and $48 \%$ for the paraboloid, $59 \%$ and $51 \%$ for the Davies-Cotton type. The absolute performances of the two designs differ only $3 \%$ at most.

In summary, our design offers good on-axis performance with adequate off-axis focusing and achieves our goal of a photon arrival time spread of 1 ns. The off-axis performance of the $7-\mathrm{m}$ reflector has been directly measured and will be reported in Section 5.2 .

\section{Spherical Mirror Facets}

Each spherical mirror facet is $0.8 \mathrm{~m}$ in diameter, $18 \mathrm{~mm}$ thick, and weighs only $5.5 \mathrm{~kg}$. The average density of the facet is about one fifth of the ordinary glass $\left(2.4-2.6 \mathrm{~g} \cdot \mathrm{cm}^{-3}\right)$, and for example, the CAT telescope employs a spherical mirror facet made of borosilicate glass which is $0.5 \mathrm{~m}$ in diameter and $10 \mathrm{~mm}$ thick [14]. A schematic cross section of a facet is shown in Figure 2. Prepregs (sheets of carbon fiber impregnated with resin) were laid on a metal mold, sandwiching a thick core of low density, high shear-strength foam to avoid twisting deformations. A sloped edge was premachined on the core to achieve a better figure after being shaped. The radius of curvature of the mold was $16.4 \mathrm{~m}$. Deflections of the facet by gravity were estimated to be as small as a few $\mu \mathrm{m}$ at the edge of a facet. A polymer sheet coated with laminated aluminum was applied on the top of these layers as the reflecting material. The facets were then sealed and cured to $120^{\circ} \mathrm{C}$ in an autoclave pressure vessel. No mechanical polishing of the surface was carried out.

Fiber patterns of the prepreg materials may make the mirror surface less smooth, and may cause a considerable amount of random scattering of incident lights. The possible scattering loss was investigated by a CCD measurement of the light focused by the CFRP mirror and by a glass mirror of good 
accuracy as a reference. Although the test was subject to non-negligible errors, it was confirmed that at least $91 \%$ of the light is focused by the CFRP mirror (without a reflectivity calibration of two materials).

We tested possible deterioration of the facets by repeating 200 cycles of a change in temperature between $0^{\circ}$ and $50^{\circ} \mathrm{C}$ in 2 hours. Changes of the humidity up to $90 \%$ were also included in the test. Within measurement errors of $0.1 \mathrm{~m}$, no change in the curvature was found after this test.

The telescope is not sheltered, so the facets are exposed to tough environmental conditions. For protection against dust, rain, and sunshine, the facets were coated with fluoride. Figure 3 shows the measured reflectivity as a function of wavelength (solid line). The reflectivity is over $80 \%$ at $340 \mathrm{~nm}-800 \mathrm{~nm}$ and falls off rather slowly to $40 \%$ at $250 \mathrm{~nm}$, corresponding both to the atmospheric transmission cut-off of Cherenkov light at $300 \mathrm{~nm}$ (the Cherenkov light spectrum after transmission [18] is shown by the dashed line) and to the response of the PMT photo-cathode with a UV-transparent window (dotted line). The reflectivity of the reflector on-site is monitored by a hand-held reflectometer at the wavelength of $480 \mathrm{~nm}[19]$. We found dust on the surface reduces the reflectivity to about $75 \%$ after several months, however, we confirmed with a sample that over a year the reflectivity repeatedly recovers easily by washing with water. The surface is free from dew until the relative humidity exceeds $83 \%$ when the wind is not strong.

Using an approximate-point light source, every spherical mirror facet was examined at the factory, and its focal length and point spread function were measured. The absolute values of the curvature radii were calibrated by the sampling measurements of three-dimensional configuration of some facets. The image size of each mirror facet was also measured on-site with a light source $5.8 \mathrm{~km}$ away. The radii of curvature of the sixty mirrors are distributed between 15.9-17.1 $\mathrm{m}$ (Figure 4), with an average of $16.4 \mathrm{~m}$ and a root-mean-square of $0.3 \mathrm{~m}$. The permissible variation was estimated by a ray-tracing calculation. The facets were arranged according to their radii of curvature from the inner 
to the outer sections of the reflector, with the shorter-curvature radii ones innermost. A CCD image of one mirror (with a distant light source) is shown in Figure 5. A typical point spread function of the sixty facets is $0^{\circ} .08$ (FWHM), and $50 \%$ and $80 \%$ of the photons are concentrated within $0^{\circ} .1$ and $0^{\circ} .15$ circles, respectively.

\section{Mirror Alignment by the Motor-Driven System}

Two watertight stepping motors are installed at the back surface of each mirror facet, and the attitude can be remotely adjusted in two perpendicular directions (Figure 6). A stainless boss at the back surface of each facet is connected to the honeycomb panel by a universal joint, and four shafts support the boss. Two shafts are driven by two stepping motors, and the other two shafts with springs firmly fix the attitude. The minimum step size corresponds to about $1 \times 10^{-4}$ degree at the focal plane, and the facets are adjustable up to \pm 3 degrees. An accuracy of $1 \times 10^{-3}$ degree is retained when motors are switched off. Facets are adjusted one by one using two motor drivers with relay switches controlled by a computer.

At the factory, the alignment of the facets was roughly adjusted to within $0^{\circ} .3$ relative to each supporting panel using a laser beam. With these alignment works, we were able to check our stepping-motor system and save on-site labor.

The alignment on-site used the $5.8 \mathrm{~km}$ distant light source at night. All mirror facets but one were covered with plastic lids, and the image of the uncovered facet was monitored on a screen at the focal plane by a CCD camera installed at the center of the dish. The attitudes of the facets were adjusted with the stepping motors using feedback information from the CCD images so that the image center lay at the focal point of the reflector.

The use of approximate-parallel light during the adjustment work may have caused a systematic shifting of the focal length of the reflector. To examine 
this, after all the sixty facets were adjusted, the effective focal length was measured with a star image. By moving the focal plane along the optical axis we surveyed for the optimum point which was found to coincide with the design focal length of $8.00 \mathrm{~m}$ within an error of $0.01 \mathrm{~m}$. As a result of the initial adjustment work, the facet axes deviated by $0^{\circ} .01-0^{\circ} .07$.

\section{Performance of the Tessellated Reflector}

The optical properties of the reflector as a whole were measured using images of several stars tracked by the telescope. Images on the focal plane screen were taken by a CCD camera at the reflector center.

\subsection{On-axis Properties}

Figure 7 shows an on-axis image of Sirius (visual magnitude of -1.5). One camera pixel $\left(0^{\circ} .115\right.$ square $)$ is superimposed for scaling. An image size of $0^{\circ} .14 \pm 0^{\circ} .01$ (FWHM) was deduced; $30 \pm 4 \%$ of the photons are concentrated in a single camera pixel, and $50 \%$ of the photons are concentrated within a circle of $0^{\circ} .16 \pm 0^{\circ} .02$ in diameter. As described in Section 1 , the characteristic difference in size between the images of gamma-rays and protons is $0^{\circ} .1^{-}$ $0^{\circ} .2$. Our optical quality meets the requirement, although we are planning to improve the alignment.

The relativistic charged particles in extensive air showers emit the Cherenkov radiation. Only high energy muons in the showers are likely to reach the ground without decaying and individual muons will radiate a characteristic Cherenkov ring (the Cherenkov threshold energy is about $4.4 \mathrm{GeV}$ ). These rings are detected in the focal plane as arcs or rings, depending on the impact parameters. A preliminary analysis of observed images shows thin muon rings with an average width of about $0^{\circ} .11[20]$. For $\sim 10 \mathrm{GeV}$ muons, the contribution of reflector aberrations to the broadening of the ring images can 
be estimated to be approximately 1.5 times the contribution of the multiple scattering in the atmosphere [21].

\subsection{Off-axis Properties}

We compared off-axis images of Sirius displacing the telescope pointing in both right ascension and declination. Some of the CCD images are synthesized and shown in Figure 8. Figure 9 shows radial point spread functions of the star displaced in right ascension by $0^{\circ} .5,1^{\circ} .0$ and $1^{\circ} .3$, respectively. Vertical scales are normalized by the peak height of the on-axis function. The light concentration within a camera pixel $\left(0^{\circ} .115\right.$ square $)$ are $28 \%, 26 \%$, and $25 \%$ (the errors are $3 \%$ for all three), compared to the on-axis value of $30 \pm 4 \%$ mentioned earlier. In comparison, the ray-tracing program predicted a relative decrease to $81 \%$ of the on-axis concentration at $1^{\circ} .3$ off axis. The peak centers were shifted as a result of the convolution of blurs of the facets and the aberrations (Section 2.2). The position of the peak center is, for example, $1^{\circ} .43$ after the scale calibration, by averaging the displacements of $1^{\circ} .3$ to the four directions.

\section{Effect of Gravitational Deformations}

At the different attitudes during observations, pointing deviations or deformation of images may appear at the focal plane due to gravitaional effects; such as deflection of the facets, of the camera stays, and of the reflector trusses. Furthermore, the facet alignment was performed while the telescope was pointed horizontally, thus the fixed setting may also have deflection to be calibrated. By tracking various stars, the effects were directly measured as the CCD images on the focal plane screen.

At elevations between $12^{\circ}$ and $85^{\circ}$ and over all the azimuthal angles, the pointing had a root-mean-square of $27^{\prime \prime}$ (Figure 10), with no dependence on elevation or azimuthal angles. 
Figure 11 shows the image sizes of nine stars as a function of the elevation angle. For elevation angles between 15-70 degrees, the images show no dependence on elevation in size within a measurement error of $0^{\circ} .01$. The deformation of the image shape is confirmed to be also negligible The fitted results of the two-dimensional point spread function of the images coincide well with each other, and the average eccentricity of the nine images is $0.99 \pm 0.01$ (statistical error only).

\section{Summary}

The new CANGAROO-II $7 \mathrm{~m}$ telescope has been constructed to observe the southern sky for very high-energy gamma-rays sources in the sub-TeV region. Observation has started with the telescope, which has a light collection area of $30 \mathrm{~m}^{2}$ and and an energy threshold of less than $300 \mathrm{GeV}$. The $f / 1.1$ paraboloid reflector offers nearly isochronous timing, and the curvature radii of facets were chosen to improve the off-axis focusing. The light-weight optical system of CFRP facets allowed considerable savings in construction labor, while the gravitational torques on the system are negligible at the focal plane. The facets are easy to handle and easy to maintain even in the open air environment. The motor-driven alignment of the tessellated reflector on-site has been shown to work well with gains in efficiency and safety. After our initial alignment was completed, the optical performance of the reflector was examined using star images. The point spread function is $0^{\circ} .14$ (FWHM), comparable in size to a camera pixel of the camera $\left(0^{\circ} .115\right.$ square). About $30 \%$ of the photons of an on-axis star image fall within a single camera pixel. The light concentration is reduced by only $18 \%$ at the edge of the $3^{\circ}$ field-of-view, confirming that off-axis aberrations are not severe for this telescope design. 


\section{Acknowledgments}

The authors thank P.G. Edwards for careful reading of the manuscript and many useful comments. The CFRP laminate mirrors have been developed in collaboration with Mitsubishi Electric Corporation, Communication Systems Center. The construction of the telescope was supported by a Grant-in-Aide in Scientific Research of the Japan Ministry of Education, Science, Sports and Cupture (No. 07247103), by the Australian Research Council, and also by the Japanese govermental fund through Institute for Cosmic Ray Research, University of Tokyo. AK, SL and TY were supported for this work by JSPS postdoctoral fellowships.

\section{References}

[1] M. Catanese and T.C. Weekes, Publ. Astron. Soc. of the Pacific vol. 111, Issue 764, (1999) 1193-1222.

[2] T.C. Weekes, Phys. Rep. 160 (1988) 1-121; R.A. Ong, Phys. Rep. 305 (1998) 93-202.

[3] R.C. Hartman et al., Astrophys. J. Suppl. 123 (1999) 79-202.

[4] R. Ong et al., in: Proc. Towards a Major Atmospheric Čerenkov Detector VI, Snowbird, 1999, in press.

[5] D.A. Smith, in: Proc. Towards a Major Atmospheric Čerenkov Detector VI, Snowbird, 1999, in press.

[6] E. Lorenz, in: Proc. Towards a Major Atmospheric Čerenkov Detector VI, Snowbird, 1999, in press.

[7] F. Krennrich, in: Proc. Towards a Major Atmospheric Čerenkov Detector VI, Snowbird, 1999, in press.

[8] W. Hofmann, in: Proc. Towards a Major Atmospheric Čerenkov Detector VI, Snowbird, 1999, in press. 
[9] T. Hara et al., Nucl. Inst. Meth. A332 (1993) 300-309; A. Kawachi, in: Proc. Int. Conf. on Neutron Stars and Pulsars, Tokyo, 1997, N. Shibazaki, N. Kawai, S. Shibata, T. Kifune (eds.), (Universal Academy Press, Inc. Tokyo) p.521-524.

[10] M. Mori et al., in: Proc. Towards a Major Atmospheric Čerenkov Detector VI, Snowbird, 1999, in press.

[11] H. Kubo et al., in: Proc. Towards a Major Atmospheric Čerenkov Detector VI, Snowbird, 1999, in press. T. Tanimori et al., in: Proc. of the 26th Int. Cosmic Ray Conf., SaltLake City, 1999, OG4.3.04.

[12] A.M. Hillas and J.R. Patterson J. Phys. G 16 (1990) 1271-1281.

[13] A. Kawachi, in: Proc. Towards a Major Atmospheric Čerenkov Detector VI, Snowbird, 1999, in press; A. Kawachi et al., in: Proc. of the 26th Int. Cosmic Ray Conf., SaltLake City, 1999, OG4.3.05.

[14] A. Barrau et al., Nucl. Inst. Meth. A416 (1998) 278-292.

[15] T. Tanimori, Y. Hayami, K. Sakurazawa, in: Proc. Towards a Major Atmospheric Cherenkov Detector IV, Padova, 1995, M. Cresti (ed.) p.316-326.

[16] D.A. Lewis, Exp. Astron. 1 (1990) 213-226.

[17] J.M. Davies, E.S. Cotton, Journal of Solar Energy 1 No.2 and 3, (1957) 16-22.

[18] K. Bernlöhr Astropart. Phys. 12 (4) (2000) 255-268.

[19] S. Dowden, J.R. Patterson, N. Wild, Meas. Sci. Technol. 8 (1997) 1258-1261.

[20] K. Okumura et al., in preparation.

[21] G. Vacanti et al., Astropart. Phys. 2, (1994) 1-11. 


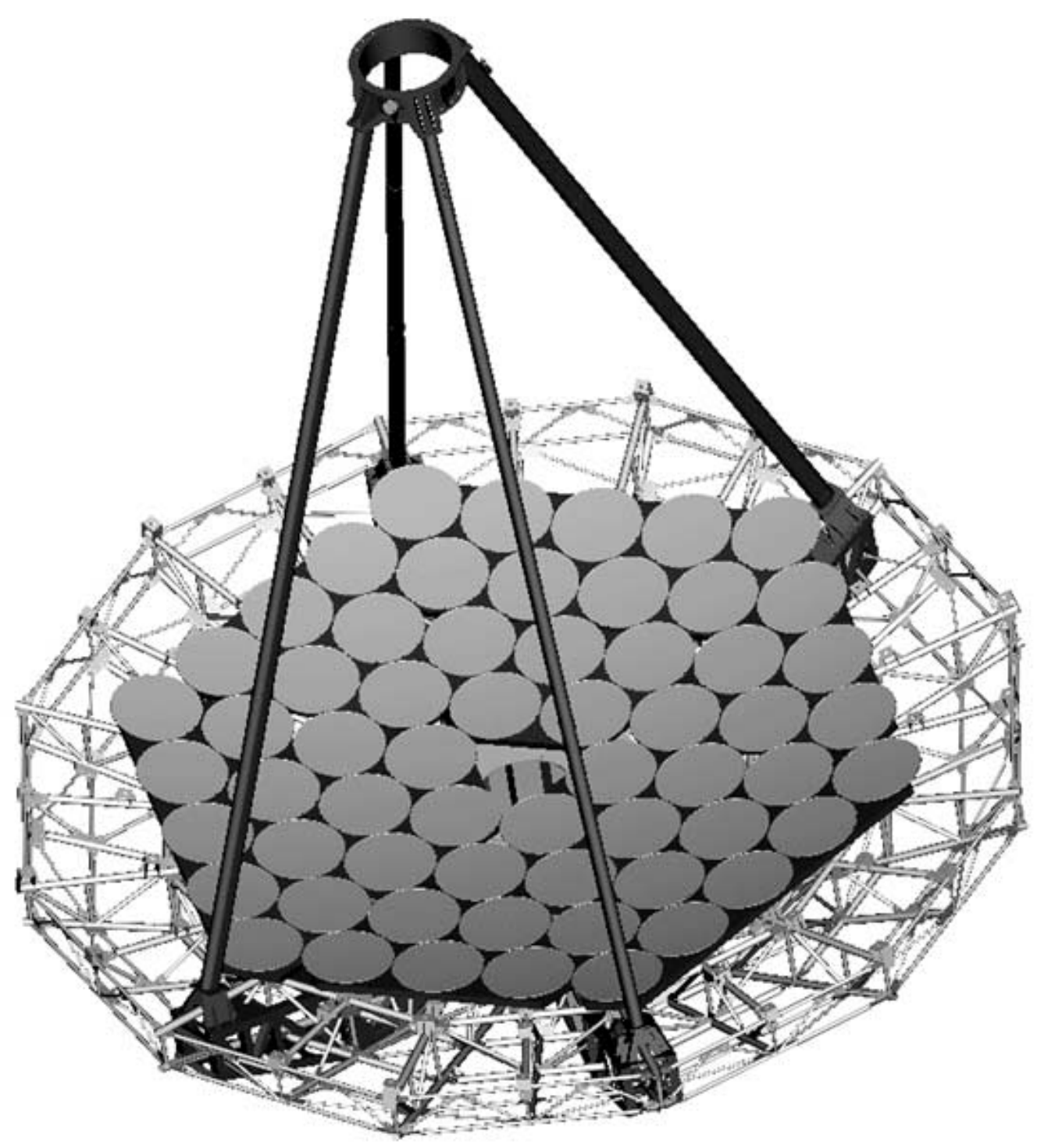

Fig. 1. A schematic drawing of the reflector and the camera support of the CANGAROO-II telescope. The paraboloid reflector is $7.2 \mathrm{~m}$ in diameter (an effective area of $30 \mathrm{~m}^{2}$ ) with an $f$-number of 1.1. The dish consists of sixty mirror facets, nine honeycomb panels for the mirror mounts, and the reflector trusses. The motor-driven system for alignment is installed under each facet. 
Al-coated polymer sheet

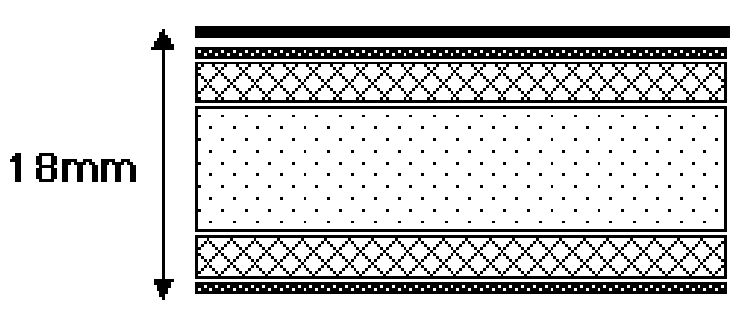
metal sheet prepregs

foam

prepregs

metal sheet

Fig. 2. A schematic illustration of the cross section of a spherical mirror facet. The "prepreg" is the intermediate composite form made by carbon fibers impregnated with resin. 


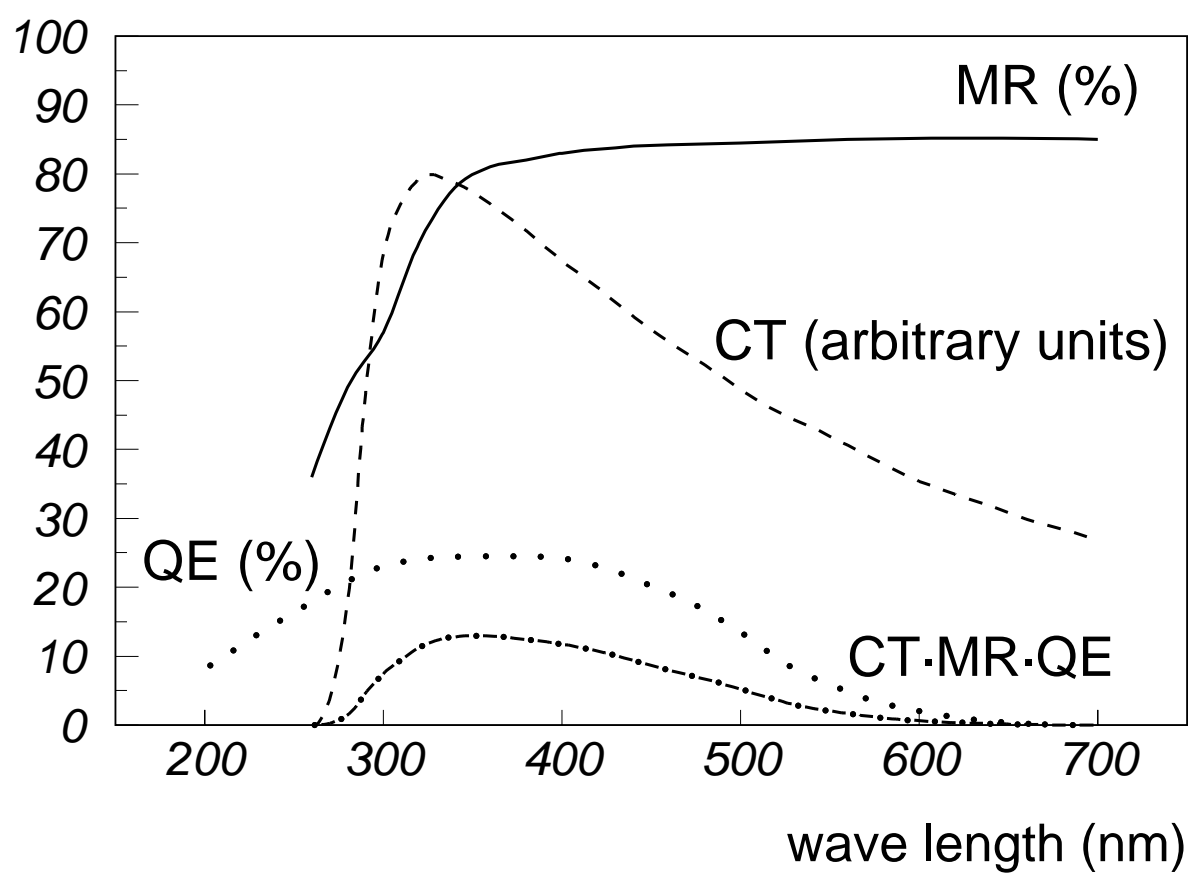

Fig. 3. The mirror reflectivity after the surface coating (MR) is shown as a function of wavelength (solid line). The quantum efficiency (QE) of R4124UV cathode (taken from the Hamamatsu catalogue) is also plotted (dotted line), together with the Cherenkov emission spectrum after atmospheric transmission (CT) in arbitrary units (dashed line). This spectrum multiplied by MR and QE (CT.MR QE) is shown as the dot-dashed line. The transmission spectrum was taken from the figure in [18]. 


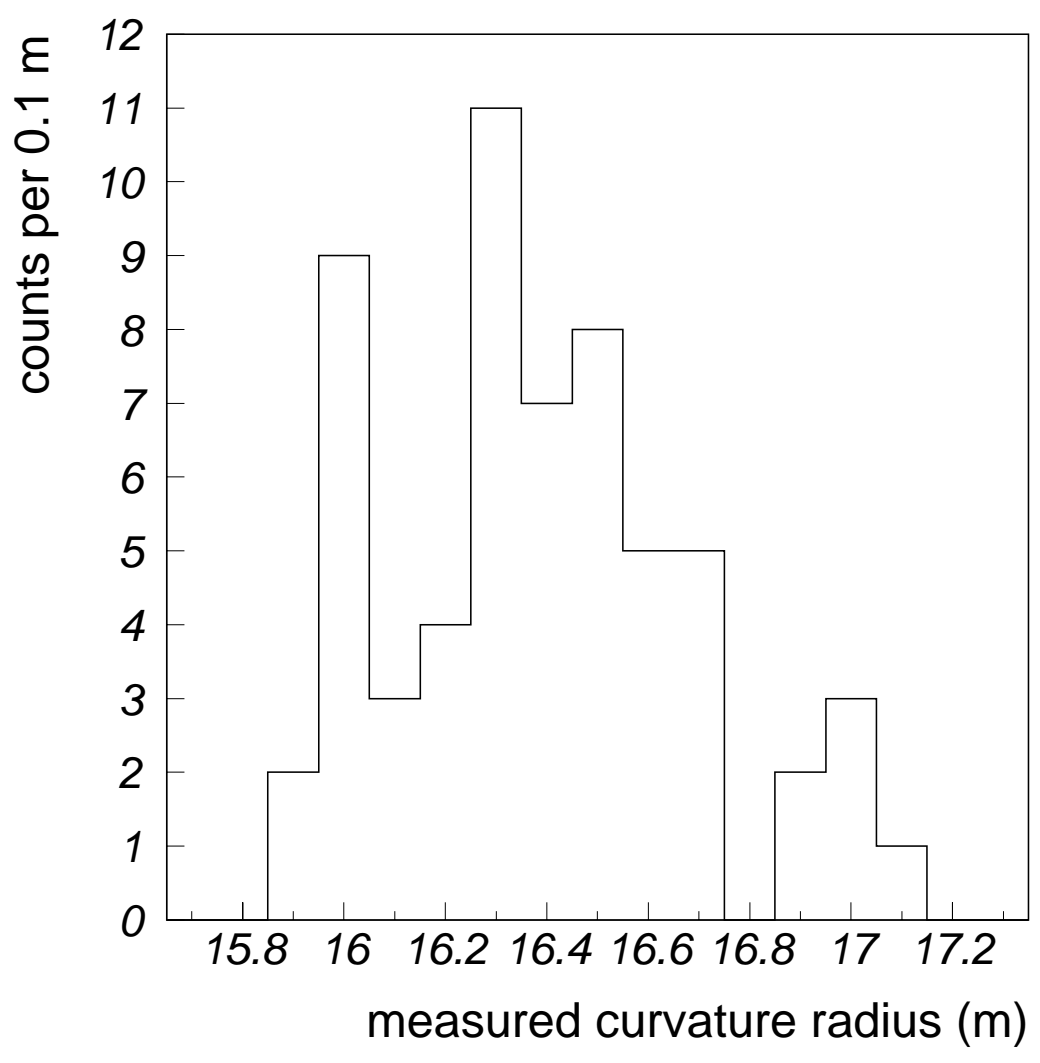

Fig. 4. The distribution of measured radii of curvature of the sixty mirror facets. The measurement error was $0.1 \mathrm{~m}$. 


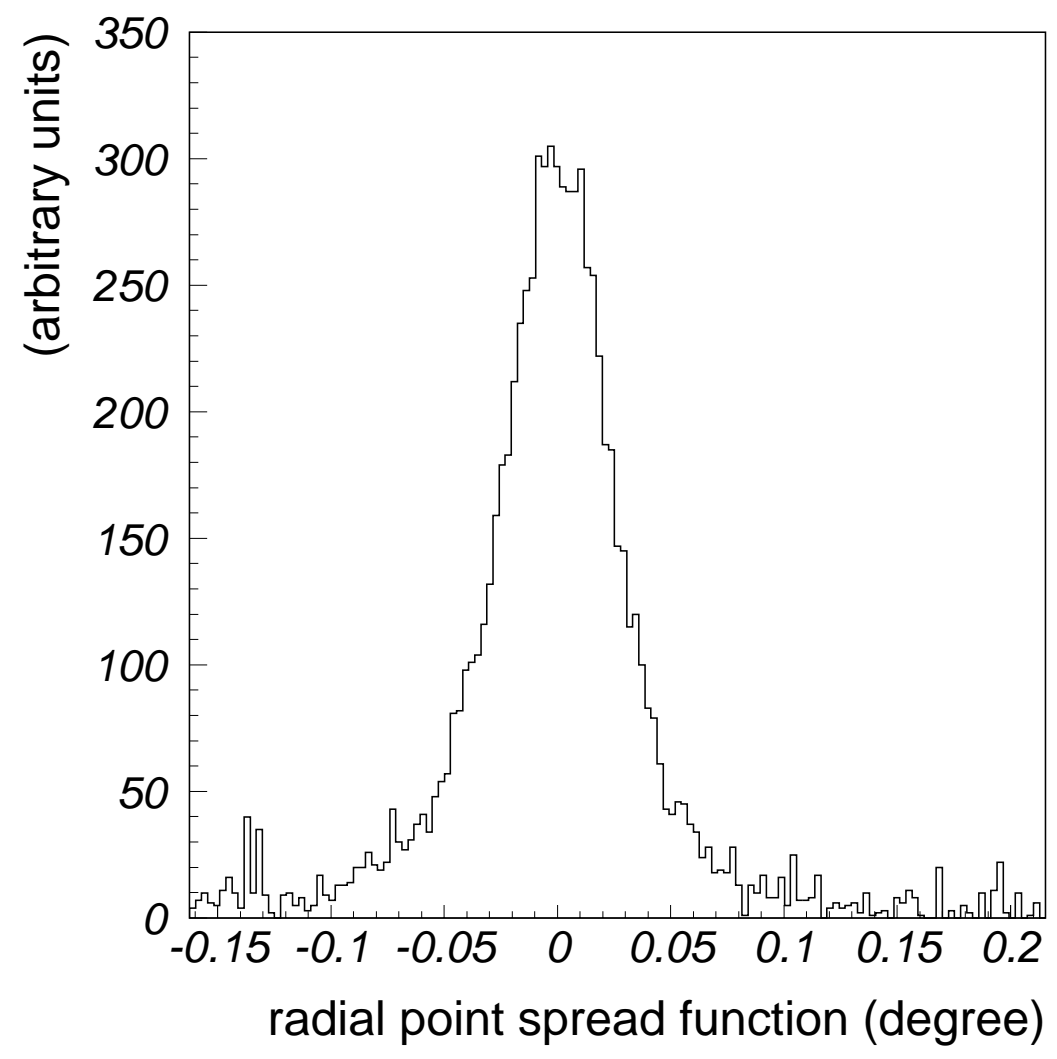

Fig. 5. A slice of the CCD image of one mirror facet with a distant light source. The radial point spread function of this mirror is $0^{\circ} .06$ (FWHM). 


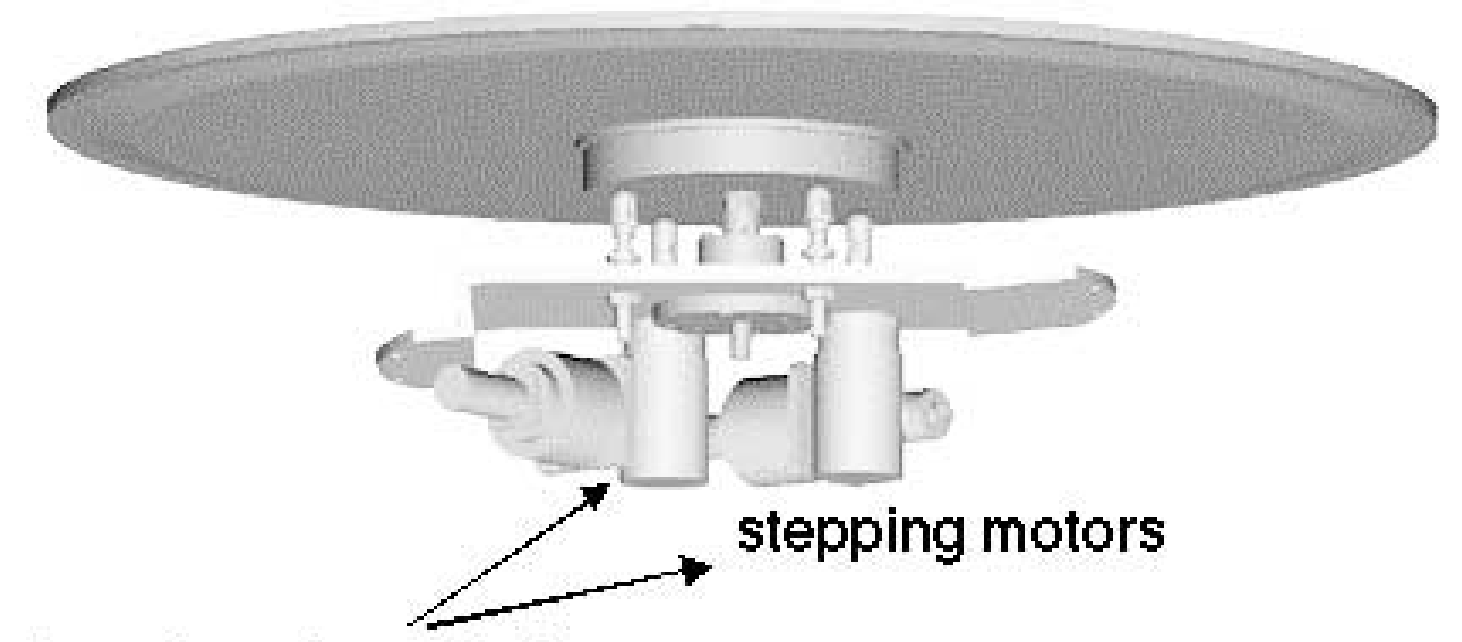

from the relay switch

Fig. 6. The motor-driven system for alignment of one mirror facet is schematically illustrated. Four shafts support a stainless boss at the back surface of each facet, where two shafts are driven by two stepping motors, and the other two shafts with springs fix the attitude. Facets are adjusted one by one using two motor drivers with relay switches controlled by a computer. 


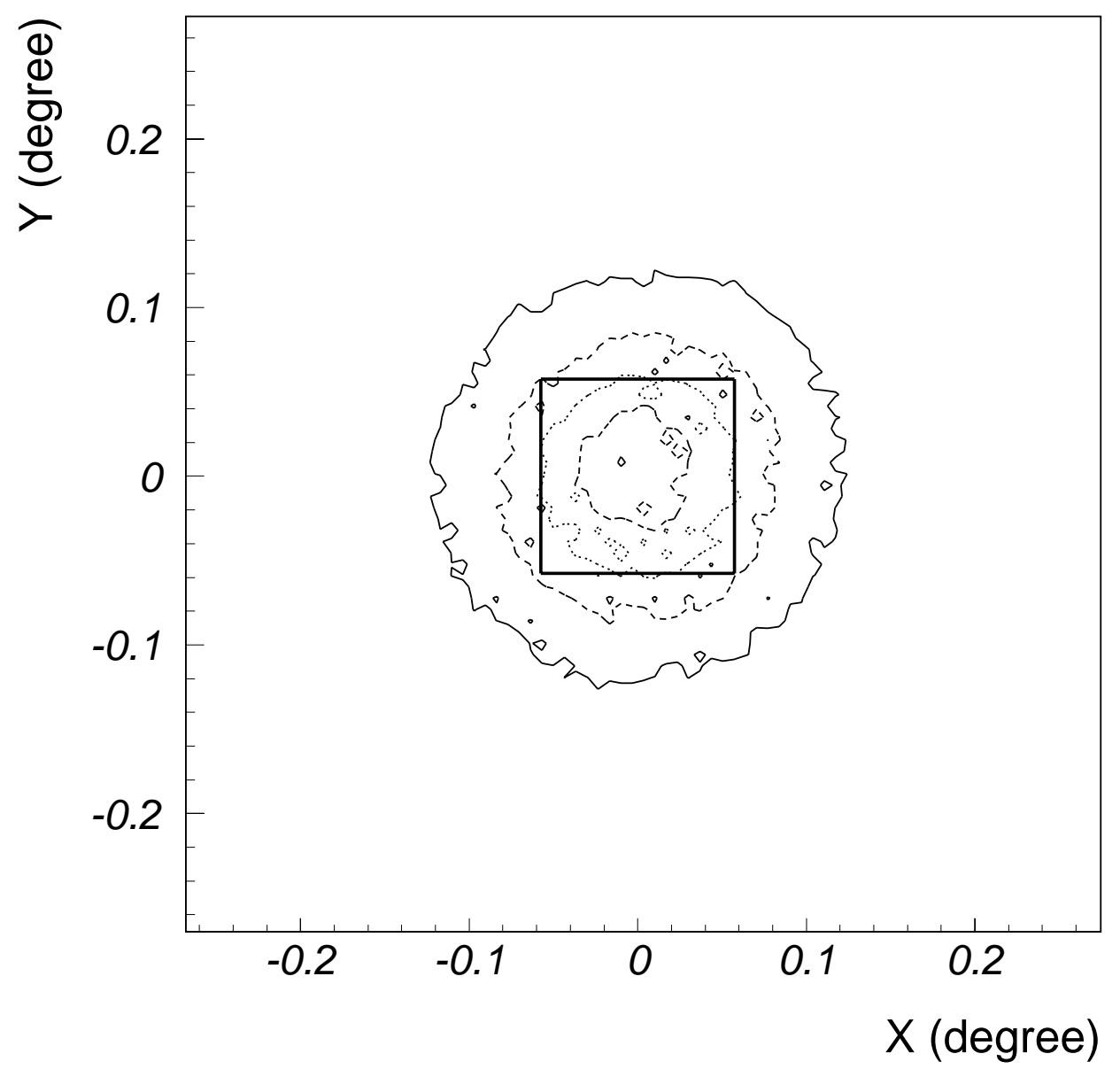

Fig. 7. A CCD image of Sirius on the optic axis. The contours are in steps of $20 \%$ of the peak intensity. A square is superimposed to show the scale of a camera pixel $\left(0^{\circ} .115\right.$ square $)$. 


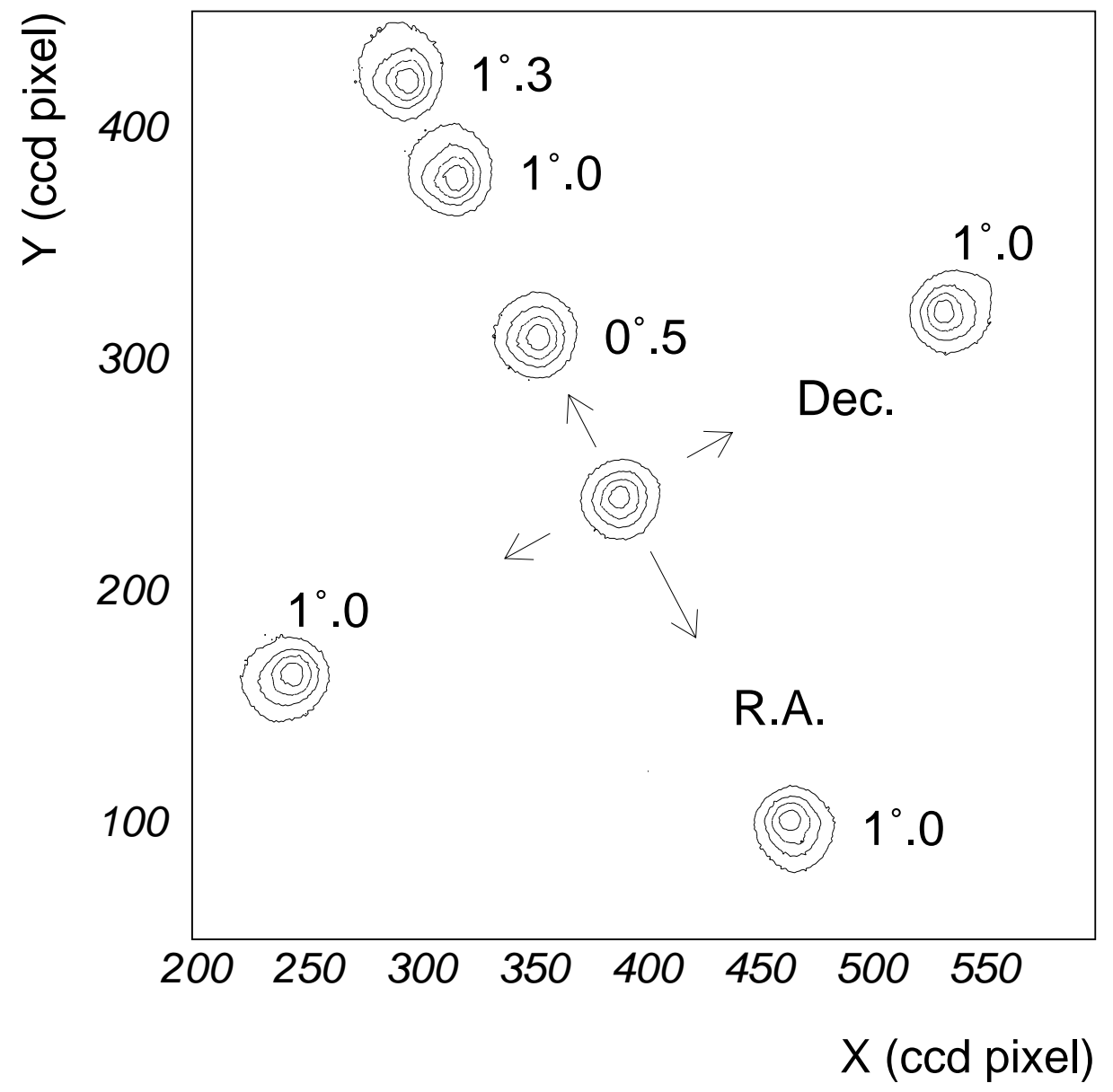

Fig. 8. A synthesized figure of some of the CCD images obtained by displacing the telescope pointing from a star. The axes are in unit of CCD pixels, corresponding to $6.7 \times 10^{-3}$ degree. The contours are in steps of $20 \%$ of the peak intensity of of each image. 


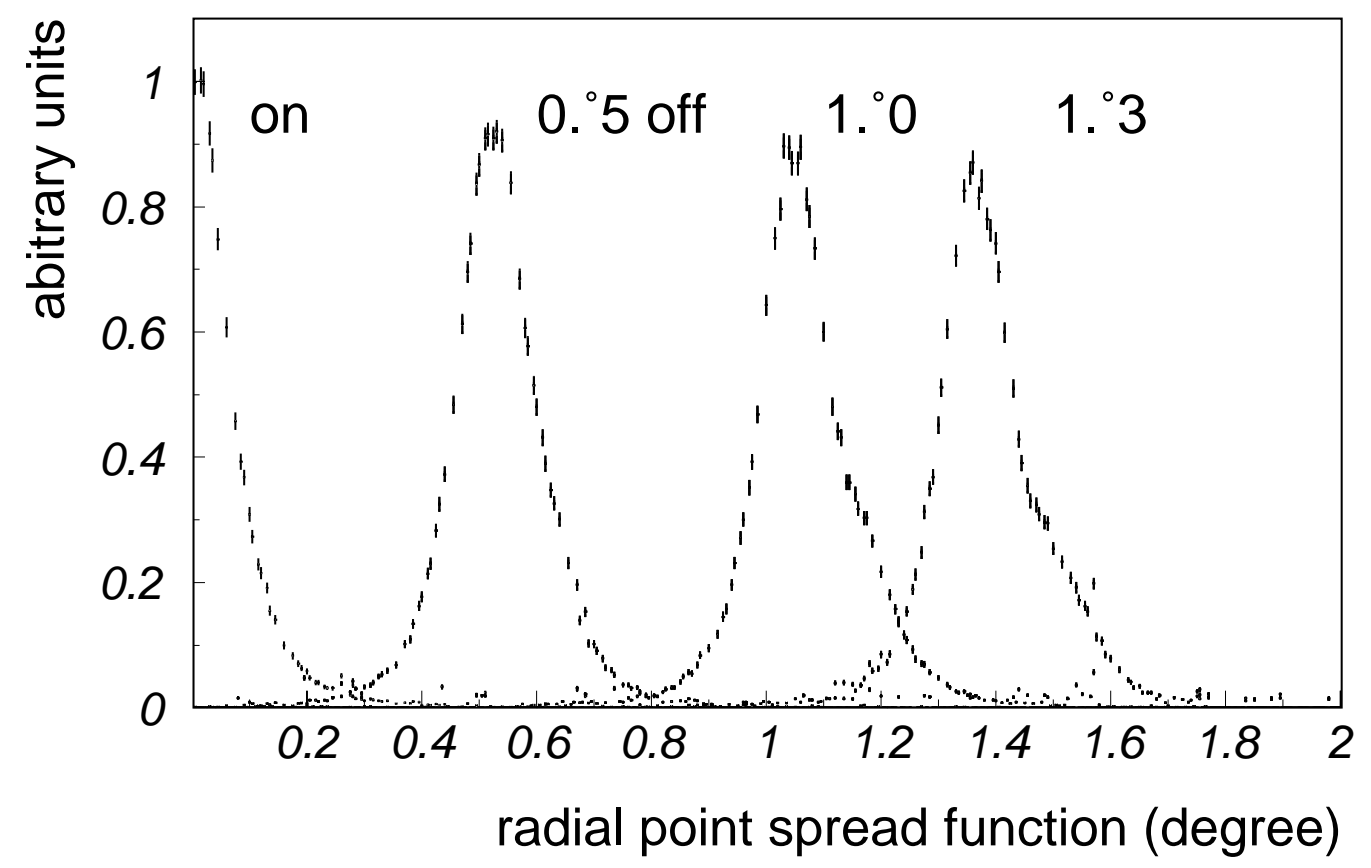

Fig. 9. Radial point spread functions at the different pointing coordinates; $(\alpha, \delta)$, $\left(\alpha-0^{\circ} .5, \delta\right)\left(\alpha-1^{\circ} .0, \delta\right)$ and $\left(\alpha-1^{\circ} .3, \delta\right)$, respectively, where $\delta=\left(-16^{\circ} 42^{\prime} 58^{\prime \prime}\right.$ $(\mathrm{J} 2000))$. Vertical scales are normalized by the peak height of the on-axis point spread function. 


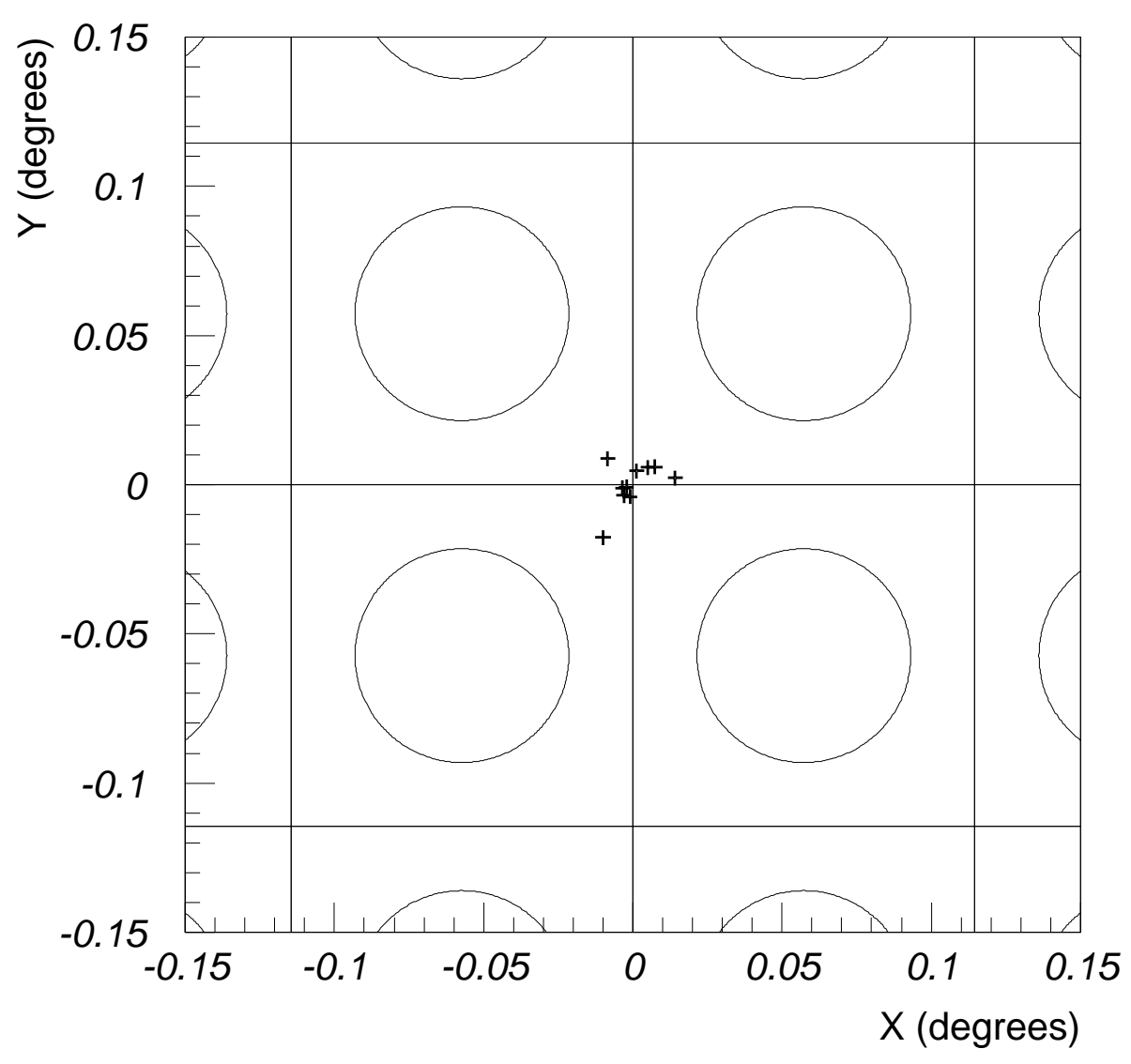

Fig. 10. A scatter plot of the center positions of the star images taken at elevations between $12^{\circ}$ and $85^{\circ}$ and over all azimuthal angles. The origin of the figure is the focal point of the telescope. An array of camera pixels $\left(0^{\circ} .115\right.$ square $)$ and of photocathode area $\left(0^{\circ} .09 \phi\right)$ of PMTs are superimposed. 


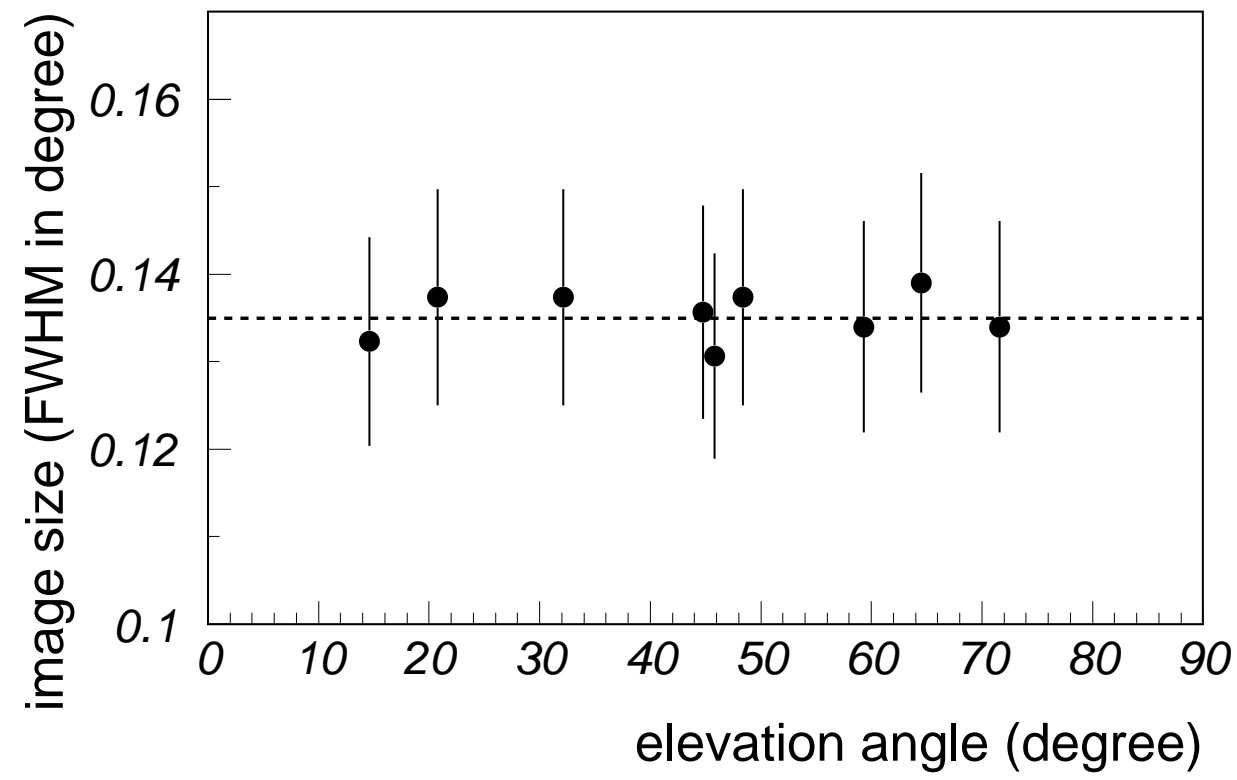

Fig. 11. Measured size (FWHM) of star images on the focal plane taken at elevation angles between $15^{\circ}$ and $70^{\circ}$. The average value $\left(0^{\circ} .135\right)$ of the stars is indicated as a dashed line. 